\title{
Purification, Characterization and Immunological Properties of the Serotype-specific Capsular Polysaccharide of Pasteurella haemolytica Serotype A7 Organisms
}

\author{
By C. ADLAM, ${ }^{*}$ J. M. KNIGHTS, ${ }^{1}$ ANNE MUGRIDGE, ${ }^{1}$ J. C. LINDON, ${ }^{2}$ \\ JANET M. WILLIAMS ${ }^{2}$ AND J. E. BEESLEY ${ }^{3}$ \\ Departments of ${ }^{1}$ Vaccine Development, ${ }^{2}$ Physical Chemistry and ${ }^{3}$ Electron Microscopy, \\ The Wellcome Research Laboratories, Beckenham, Kent BR3 3BS, UK
}

(Received 23 September 1985)

\begin{abstract}
The serotype-specific capsular polysaccharide from two strains of Pasteurella haemolytica serotype $\mathrm{A} 7$ organisms was purified and characterized by chemical analysis and by ${ }^{1} \mathrm{H}$ and ${ }^{13} \mathrm{C}$ NMR spectroscopy using one- and two-dimensional methods. The polymer has the repeating unit $\rightarrow 3$ - $\beta$-2-acetamido-2-deoxygalactopyranose-( $\rightarrow 3)$ - $\alpha$-2-acetamido-2-deoxy-6-O-acetylglucopyranose-(1-phosphate $\rightarrow$. It was immunogenic (capable of eliciting antibodies) for sheep. Chemical removal of $O$-acetyl groups destroyed both the ability of the polymer to adhere to sheep erythrocytes at neutral $\mathrm{pH}$ and the ability to form immune precipitates with specific antisera. Studies using the protein A-gold technique in the electron microscope showed the polysaccharide to be peripherally localized on the bacterial surface.
\end{abstract}

\section{INTRODUCTION}

Pasteurella haemolytica organisms are responsible for pneumonia and septicaemia of sheep and cattle. They are separable into 15 different serotypes belonging to two different biotypes, $\mathrm{A}$ and $\mathrm{T}$. In an attempt to understand more about possible virulence factors of these organisms, we have previously purified and characterized the capsular polysaccharides of three of these serotypes (Adlam et al., 1984, 1985a, b). This paper reports the structure and some immunological properties of the polysaccharide from serotype A7.

\section{METHODS}

Bacterial strains and growth conditions. The two A7 serotype strains used were maintained as freeze dried cultures and grown in Pasteurella broth no. 7 (Wellcome). Strain CN5498 was originally obtained from the Moredun Institute, Edinburgh, UK, as strain H1/A. Strain CN7318 was isolated from a pneumonic lamb at the Wellcome Research Laboratories. The majority of studies were done with strain CN7318 grown in a 2001 fermenter as previously described (Adlam et al., 1984). Confirmatory studies were done with strain CN5498 grown in 21 conical shake flasks $\left(1.51\right.$ medium, $37^{\circ} \mathrm{C}, 24 \mathrm{~h}, 140 \mathrm{r} . \mathrm{p} . \mathrm{m}$.). The $\mathrm{OD}_{650} 1 \mathrm{~m}$ of the final organism suspension was 6.0 for CN7318 and 1.9 for CN5498.

Other preparative, analytical and immunological methods. Methods for the preparation of crude and highly purified polysaccharides, chemical analysis, de- $O$-acetylation, ninhydrin degradation, amino acid analysis, molecular mass determination, production of antiserum, immunodiffusion, passive haemagglutination, toxicity testing, electron microscopy and high pressure liquid chromatography (HPLC) were as previously described (Adlam et al., 1984; 1985a, b).

Nuclear magnetic resonance (NMR) spectroscopy. Spectra were measured as $\mathrm{D}_{2} \mathrm{O}$ solutions using a Bruker WM360 instrument operating in the pulse-Fourier transform mode at ambient temperature (approximately $25^{\circ} \mathrm{C}$ for ${ }^{1} \mathrm{H}$ and $40^{\circ} \mathrm{C}$ for ${ }^{13} \mathrm{C}$ ).

${ }^{1} \mathrm{H}$ NMR spectra were measured at $360 \cdot 13 \mathrm{MHz}$ and ${ }^{13} \mathrm{C}$ NMR spectra were obtained at $90 \cdot 5 \mathrm{MHz} \cdot{ }^{1} \mathrm{H}$ and ${ }^{13} \mathrm{C}$ chemical shifts were referenced to internal acetone at $2.225 \delta$ and external dioxan at 67.4 p.p.m. respectively. For the two-dimensional ${ }^{13} \mathrm{C}-1 \mathrm{H}$ correlation experiment (Bodenhausen \& Freeman, 1977) purified polymer (60 mg) 
was dissolved in $0.4 \mathrm{ml} \mathrm{D}_{2} \mathrm{O}$ and the spectrum measured at $26^{\circ} \mathrm{C}$. Each acquisition using the HCCORR program from the Bruker Aspect 2000 program library was into $2 \mathrm{~K}$ data points over a spectral width of $5376 \mathrm{~Hz}$. Two hundred and fifty six different time increments were used thus defining 256 data points zero filled to 512 points in the $\mathrm{Fl}$ dimension. The $90^{\circ}$ pulse widths for ${ }^{1} \mathrm{H}$ and ${ }^{13} \mathrm{C}$ were $28.0 \mu$ s and $7.0 \mu$ s respectively. Six hundred transients were acquired at each time increment. Lorentzian-Gaussian resolution enhancement was used in the Fl domain. For ${ }^{1} \mathrm{H}^{-1} \mathrm{H}$ two-dimensional COSY experiments (homo-nuclear coupling constant correlation; Bax \& Freeman, 1981 ) a solution in $\mathrm{D}_{2} \mathrm{O}$ at about $40 \mathrm{mg} \mathrm{ml}^{-1}$ was used. $1 \mathrm{~K}$ time domain points were acquired for 256 different time intervals, the final data matrix being $1 \mathrm{~K}$ by 512 points. The F2 spectral width was $729.9 \mathrm{~Hz}$. Sine bell apodization was applied in both domains before Fourier transformation; 440 scans at each time increment were averaged. The ${ }^{1} \mathrm{H} 90^{\circ}$ pulse width was $5.8 \mu \mathrm{s}$.

\section{RESULTS}

\section{Yield of extract antigens}

The yield of crude acetone precipitate per litre of culture supernatant was $0.64 \mathrm{~g}$ for CN7318. When this was further purified, the yield of purified polymer was $36.9 \%$. No attempt was made to optimize yield of antigen by altering bacterial growth conditions.

\section{Physical and chemical characteristics}

The purified polymer was a white powder, freely soluble in water. On passage through a Sepharose $2 \mathrm{~B}$ column a single $206 \mathrm{~nm}$ absorbing peak of molecular mass approximately 1.9 $\mathrm{MDa}$ was observed. Chemical analysis showed the presence of phosphate $(14.4 \%)$, hexosamines $(75.2 \%$; with reference to an equal mixture of 2-amino-2-deoxyglucose and 2 amino-2-deoxygalactose) and $O$-acetyl groups $(11.1 \%$, with reference to an acetylcholine chloride standard). Assays for hexuronic acids, sialic acids, hexoses and protein gave essentially negative results. The optical properties of the component sugars in the polymer were not examined.

Attempts to remove $O$-acetyl groups from the purified polymer using $0.1 \mathrm{M}-\mathrm{NaOH}$ (Bhattacharjee et al., 1975) resulted in complete destruction of the polymer. Successful de-Oacetylation was, however, achieved using $0 \cdot 02 \mathrm{M}-\mathrm{NaOH}$.

\section{Amino acid analysis}

A sample of purified polymer prepared from CN7318 and hydrolysed with $6.0 \mathrm{M}-\mathrm{HCl}$ for $18 \mathrm{~h}$ at $100{ }^{\circ} \mathrm{C}$ gave (apart from ammonia, which was the last peak to elute) two major peaks of approximately equal area when analysed on an amino acid analyser. These peaks eluted at times identical to those given by 2-amino-2-deoxyglucose and 2-amino-2-deoxygalactose standards and were separable from a 2-amino-2-deoxymannose standard peak.

\section{Ninhydrin degradation}

Thin layer chromatographic analysis of the ninhydrin degradation products of the acid hydrolysed polymer yielded two purple staining spots which had $R_{F}$ values identical to those of arabinose $(0.51)$ and lyxose $(0.58)$ standards. These two sugars were the expected degradation products from 2-amino-2-deoxyglucose and 2-amino-2-deoxygalactose (Stoffyn \& Jeanloz, 1954).

\section{NMR spectroscopy}

(a) The basic structure. The proton decoupled ${ }^{13} \mathrm{C}$ NMR spectrum of the native material (Fig. 1) showed resonances (both $\mathrm{C}=\mathrm{O}$ and $\mathrm{CH}_{3}$ ) typical of two $\mathrm{N}$-acetyl groups, one $\mathrm{O}$-acetyl and 12 resonances in the normal sugar region (50-110 p.p.m.). The relative intensities of the carbohydrate resonances confirmed that the two hexosamines were equimolar. The general pattern of chemical shifts showed both sugars to be $N$-acetylated, to be pyranose forms and that one sugar was uniquely $O$-acetylated at $\mathrm{C} 6$ because of the distinctive shifts in the 60-65 p.p.m. region. The other sugar had a free $\mathrm{C} 6 \mathrm{CH}_{2} \mathrm{OH}$ residue. The two anomeric carbon signals appeared at 102 and 95 p.p.m., typical of one $\alpha$-linked and one $\beta$-linked sugar. This was confirmed by the ${ }^{1} \mathrm{H}$ chemical shifts of the anomeric protons at $5 \cdot 3 \delta$ and $4.6 \delta$, also typical for $\alpha$ hexoses and $\beta$-hexoses respectively. The ${ }^{13} \mathrm{C}$ spectrum of the de- $O$-acetylated polysaccharide is 


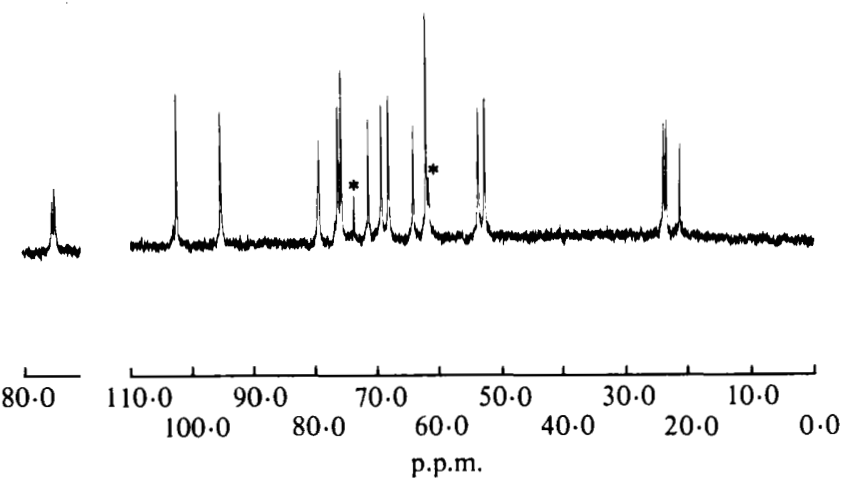

Fig. 1. ${ }^{13} \mathrm{C}$ NMR of $\mathrm{A} 7$ native polysaccharide in $\mathrm{D}_{2} \mathrm{O}$ (strain $\left.\mathrm{CN} 5498\right)$. (* $\mathrm{C} 5, \mathrm{C} 6$ of de-O-acetylated polysaccharide).

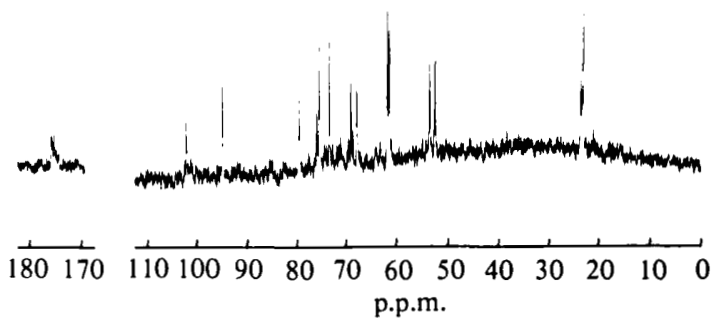

Fig. 2. ${ }^{13} \mathrm{C}$ NMR of $\mathrm{A} 7$ de-O-acetylated polysaccharide in $\mathrm{D}_{2} \mathrm{O}$ (strain $\mathrm{CN} 7318$ ).

shown in Fig. 2. In this case four resonances showed spin coupling to ${ }^{31} \mathrm{P}$. These were the $\alpha$-sugar $\mathrm{C} 1$ resonance, both $\mathrm{C} 2$ resonances (easily identified at 50-55 p.p.m.) and one other in the normal sugar region. These couplings observed only over two or three bonds indicated that the phosphate was present as a phophodiester and was linked to the $\mathrm{Cl}$ of the $\alpha$-sugar and to the $\mathrm{C} 3$ of the $\beta$-sugar.

The information available at this stage enabled the number of possible repeating units to be reduced to four straight chain and two branched chain structures (ignoring which $\mathrm{C} 6$ was $O$ acetylated), viz.:

$$
\begin{gathered}
3) \rightarrow \alpha \text {-GalNAc- }\left(1-\mathrm{PO}_{4}-3\right)-\beta-\text { GlcNAc } \rightarrow(1 \\
4) \rightarrow \alpha \text {-GalNAc- }\left(1-\mathrm{PO}_{4}-3\right)-\beta-\text { GlcNAc } \rightarrow(1 \\
3) \rightarrow \alpha \text {-GlcNAc- }\left(1-\mathrm{PO}_{4}-3\right)-\beta \text {-GalNAc } \rightarrow(1 \\
4) \rightarrow \alpha \text {-GlcNAc- }\left(1-\mathrm{PO}_{4}-3\right)-\beta \text {-GalNAc } \rightarrow(1 \\
4) \beta \text {-GalNAc- }(\mathrm{I} \\
3 \\
\uparrow \\
\mathrm{PO}_{4} \\
\uparrow \\
1 \\
\alpha-\text { GlcNAc }
\end{gathered}
$$

4) $\beta$-GlcNAc-(1

$$
\begin{gathered}
3 \\
\uparrow \\
\mathrm{PO}_{4} \\
\uparrow \\
\alpha \text {-GalNAc }
\end{gathered}
$$



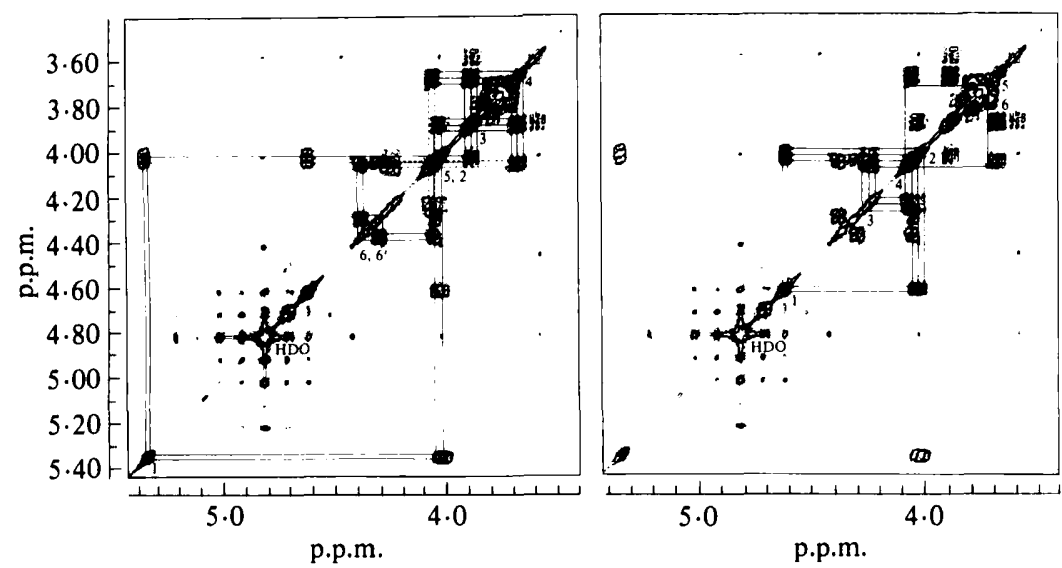

Fig. 3. Contour plot of ${ }^{\prime} \mathrm{H}-{ }^{\prime} \mathrm{H}$ two-dimensional COSY experiment on the native polysaccharide (strain CN 5498) showing connectivities at left for protons of $\alpha$-GlcNAc and at right for $\beta$-GalNAc. Peaks from de- $O$-acetylated polysaccharide are not indicated. To avoid confusion, not all connectivity lines are shown from $6,6^{\prime}$ to 5 in $\alpha$-GlcNAc.
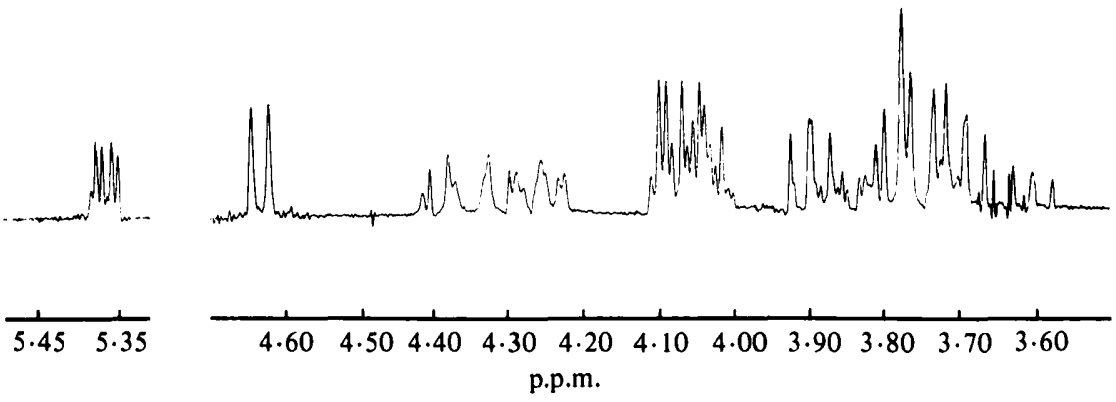

Fig. 4. ' $\mathrm{H}$ NMR of $\mathrm{A} 7$ native polysaccharide in $\mathrm{D}_{2} \mathrm{O}$ (strain $\mathrm{CN} 5498$ ); resonances from de- $O$ acetylated polysaccharide are not indicated.

(b) Identification of the $\alpha$-and $\beta$-sugars. This was achieved by a thorough analysis of the ${ }^{1} \mathrm{H}$ spectrum of the native polysaccharide by a combination of one-dimensional decoupling and two-dimensional coupling correlation experiments (COSY) to map out the J-coupling connectivities and their magnitudes. The differentiation of galacto- and gluco- configurations lies in the values of $\mathrm{J}(\mathrm{H} 3-\mathrm{H} 4)$ which for the former is small and of an axial-equatorial nature and for the latter is large and of an axial-axial type. The two-dimensional COSY spectrum of the native polysaccharide is shown in Fig. 3 as a contour plot. The normal spectrum appears along the diagonal with off-diagonal peaks connecting resonances which are J-coupled at each other. The fine structure of the off-diagonal peaks allows identification of the number and magnitude of couplings (including those to ${ }^{31} \mathrm{P}$ ).

Analysis of the two-dimensional spectrum was started at resonances which had been assigned in the one-dimensional ${ }^{1} \mathrm{H}$ spectrum. (Fig. 4). Thus the $\beta$ anomeric proton $\mathrm{Hl}$ at $\delta 4 \cdot 6$, which was a doublet $(\mathrm{J} \sim 10 \mathrm{~Hz})$ in the normal one-dimensional spectrum, was connected to $\mathrm{H} 2$ via a pair of off-diagonal peaks with $(2 \times 3)$ multiplet fine structure. The connecting lines from this pair of off-diagonal peaks to the spectrum on the diagonal showed $\mathrm{Hl}$ to be a doublet $(\mathrm{J} \sim 10 \mathrm{~Hz})$ as already known and $\mathrm{H} 2$ to be a triplet $(\mathrm{J} \sim 10 \mathrm{~Hz})$ at $\sim \delta 4.03$. This would be expected for $\mathrm{H} 2$ for either $\beta$-glucosamine or $\beta$-galactosamine. $\mathrm{H} 2$ was also coupled to a proton resonance at $\delta 4 \cdot 24$, i.e. $\mathrm{H} 3$, the fine structure of the off-diagonal peak linking them being $(3 \times 3)$ showing $\mathrm{H} 3 \beta$ to be a large triplet $(\mathrm{J} \sim 10 \mathrm{~Hz})$. This $\mathrm{H} 3 \beta$ resonance was also coupled to a resonance at $\delta 3 \cdot 9$, i.e. $\mathrm{H} 4 \beta$, the pair of off-diagonal peaks linking them being $(3 \times 1)$ multiplicity showing a triplet at $\mathrm{H} 3$ and a coupled singlet or a coupling less than the limit of resolution $(\sim 5 \mathrm{~Hz})$ at $\mathrm{H} 4$. 
Table 1. 'H NMR parameters for the native polysaccharide (strain 5498)

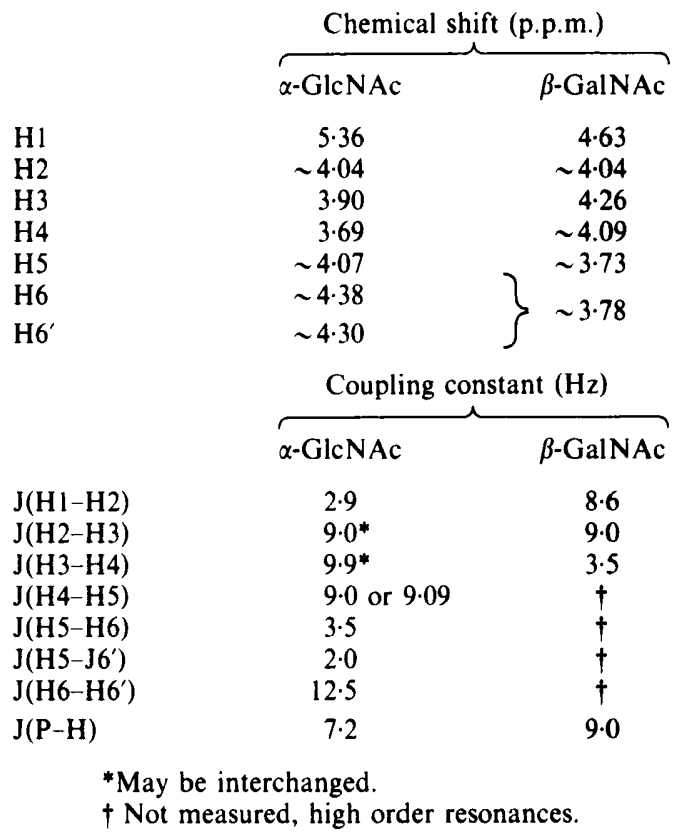

The two large couplings at $\mathrm{H} 3$ may therefore be explained by coupling of $\mathrm{H} 3$ to $\mathrm{H} 2$, already established, and to ${ }^{31} \mathrm{P}$ since no large coupling was present in the $\mathrm{H} 4$ resonance. This agreed with the deductions made from the ${ }^{13} \mathrm{C}$ NMR that phosphodiester was substituted at $\mathrm{C} 3$ in the $\beta$-hexose. The small coupling between $\mathrm{H} 3 \beta$ and $\mathrm{H} 4 \beta$ established the $\beta$-hexose as $\beta$-GalNAc. A one-dimensional spin decoupling experiment irradiating at $\mathrm{H} 4$ and then at $\mathrm{H} 3$ confirmed $\mathrm{J}_{3,4} \sim$ $3 \mathrm{~Hz}$ in the $\beta$-hexose which is typical for this sugar.

Analysing the two-dimensional COSY spectrum for the $\alpha$-hexose in a similar way showed $\mathrm{H} 2 \alpha$ to be very close to $\mathrm{H} 2 \beta(\delta 4.03)$. Connectivity peaks of $(3 \times 2)$ multiplicity were observed between $\mathrm{H} 2$ and the resolved one proton triplet resonance at $(\sim \delta 3.88, \mathrm{~J} \sim 10 \mathrm{~Hz})$ showing this to be from $\mathrm{H} 3$. This in turn was connected to $\mathrm{H} 4$ at $\delta 3.67$ via a pair of off-diagonal peaks of multiplicity $(3 \times 3)$ showing both $\mathrm{H} 3 \alpha$ and $\mathrm{H} 4 \alpha$ resonance to be triplets $(\mathrm{J} \sim 9.5 \mathrm{~Hz})$. This confirmed that the three protons $\mathrm{H} 3, \mathrm{H} 4$ and $\mathrm{H} 5$ of the $\alpha$-hexose were all axial and thus that $\alpha$ GlcNAc was present.

Other ${ }^{1} \mathrm{H}$ chemical shifts, such as $\mathrm{H} 5$ and both $\mathrm{H} 6$ hydrogens could be deduced in a similar fashion and all ${ }^{1} \mathrm{H}$ NMR parameters which have been extracted are shown in Table 1 . The detailed connectivity patterns for the $\alpha$-GlcNAc and the $\beta$-GalNAc resonances are shown separately in Fig. 3.

At this stage only three repeating unit structures were consistent with the data, i.e. III, IV, V, and differentiation between these rested on the known substituent effects (e.g. phosphorylation, glycosidation) on ${ }^{13} \mathrm{C}$ chemical shifts of carbohydrates. This in turn required a full assignment of the ${ }^{13} \mathrm{C}$ NMR spectrum.

(c) ${ }^{13} \mathrm{C}$ spectrum assignment and deduction of the repeating unit. The assignment of the ${ }^{13} \mathrm{C}$ spectrum could now proceed using a two-dimensional experiment which correlated ${ }^{13} \mathrm{C}$ and ${ }^{1} \mathrm{H}$ chemical shifts via their mutual one bond coupling constant. This was possible because of the almost total assignment of the ${ }^{1} \mathrm{H}$ spectrum using the COSY technique above. This ${ }^{1} \mathrm{H}-{ }^{13} \mathrm{C}$ correlation spectrum is shown in Fig. 5 for the native polysaccharide. From a knowledge of the ${ }^{1} \mathrm{H}$ chemical shifts for each sugar, the corresponding ${ }^{13} \mathrm{C}$ chemical shifts could be read off and these are given in Table 2 with their assignments. 


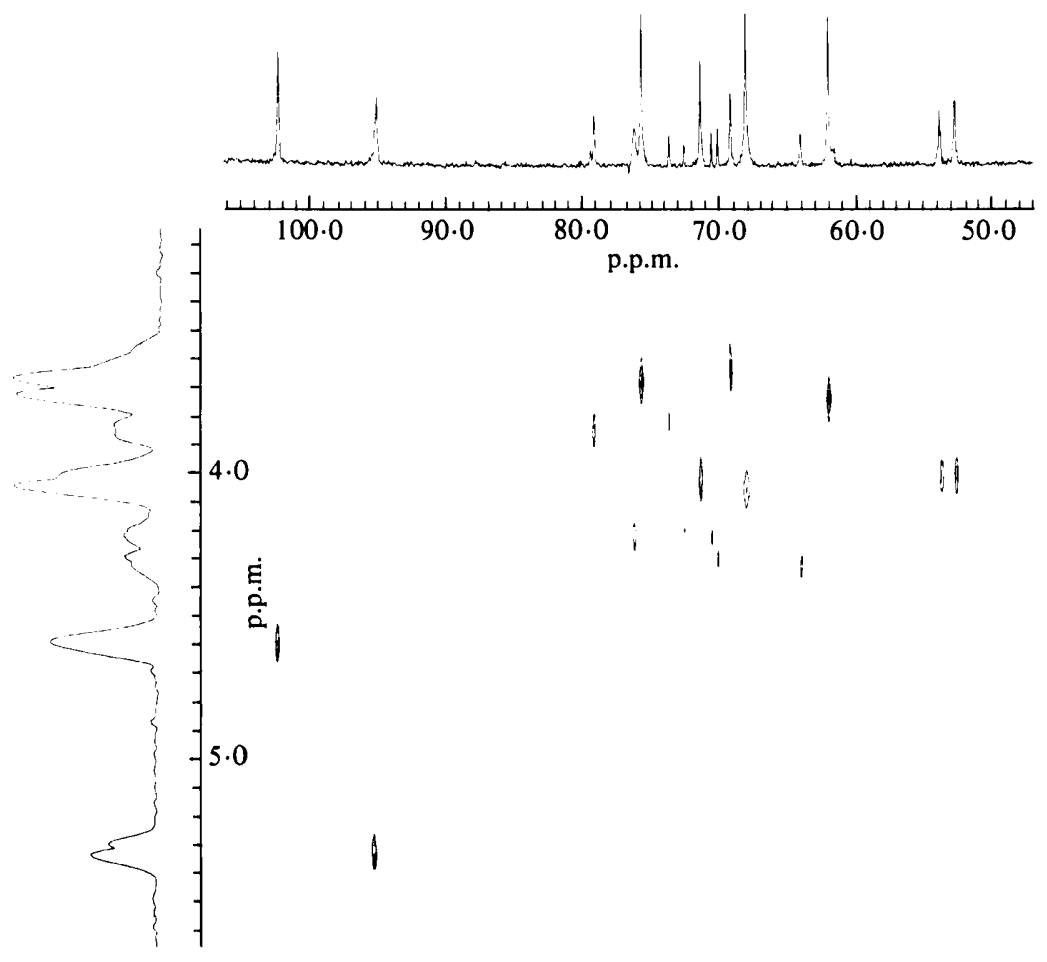

Fig. 5. Contour plot of $a^{13} \mathrm{C}-1{ }^{1} \mathrm{H}$ correlation experiment on the native polysaccharide (strain CN5498).

Distinction between structures III, IV or V relied on predicting the ${ }^{13} \mathrm{C}$ chemical shifts for

$$
\begin{aligned}
& \text { 3) } \rightarrow \alpha-\text { GlcNAc- }\left(1-\mathrm{PO}_{4}^{-}\right. \\
& \text {4) } \rightarrow \alpha-\text { GlcNAc- }\left(1-\mathrm{PO}_{4}-\right. \\
& \alpha \text {-GlcNAc-(1-PO }{ }_{4}^{-}
\end{aligned}
$$

and comparing them with the data in Table 2. The C3 chemical shift of the $\alpha$-GlcNAc residue occurred at approximately 5 p.p.m. to low field of that in $\alpha$-GlcNAc l-phosphate (Bundle et al., 1973) indicating further substitution at C3 and hence showing Structure III to be the basic repeating unit. This arrangement of -3$) \alpha-G l c N A c-\left(1-P_{4}\right.$ - also occurs in the polysaccharide from Neisseria meningitidis serotype L (Jennings et al., 1983), the data on which is included in Table 2, showing good agreement.

(d) Position of $O$-acetylation. The ${ }^{1} \mathrm{H} C O S Y$ experiment had already served to assign the $\mathrm{H} 6$ hydrogens of both sugars (see Figs 3 and 4). Knowing these ${ }^{1} \mathrm{H}$ shifts, the ${ }^{13} \mathrm{C}-{ }^{1} \mathrm{H}$ correlation experiment showed that for the $\alpha$-GlcNAc the $\mathrm{H} 6$ hydrogens were correlated with the $\mathrm{C} 6$ resonance at 63.8 p.p.m. indicating $O$-acetylation at this residue. The $\beta$-GalNAc H6 hydrogens were correlated with the $\mathrm{C} 6$ resonances at 62.0 p.p.m. indicating a free $\mathrm{CH}_{2} \mathrm{OH}$ group.

(e) Interpretation of minor resonance. In native polysaccharide purified from both bacterial strains, minor resonances were observed in the ${ }^{13} \mathrm{C} \mathrm{NMR}$ (Fig. 1) at $\sim 74$ p.p.m. and $\sim 62$ p.p.m.; these chemical shifts correspond to those of $\mathrm{C} 5$ and $\mathrm{C} 6$ respectively of the de- $O$ acetylated polysaccharide. No other minor signals were observed. This observation is compatible with some de- $O$-acetylated polysaccharide being present in the samples because the substituent effect of $O$-acetyl is only expected to be signified at $\alpha$ (i.e. C6) and $\beta$ (i.e. C5) positions. Comparison of line intensities showed the degree of $O$-acetylation to be $75-80 \%$ in the native polysaccharide in both strains examined.

In the ${ }^{1} \mathrm{H}$ NMR spectrum of the native polysaccharide (Fig. 4) minor signals were also observed. Following the assignment in the ${ }^{13} \mathrm{C}$ NMR these minor proton signals were assigned 


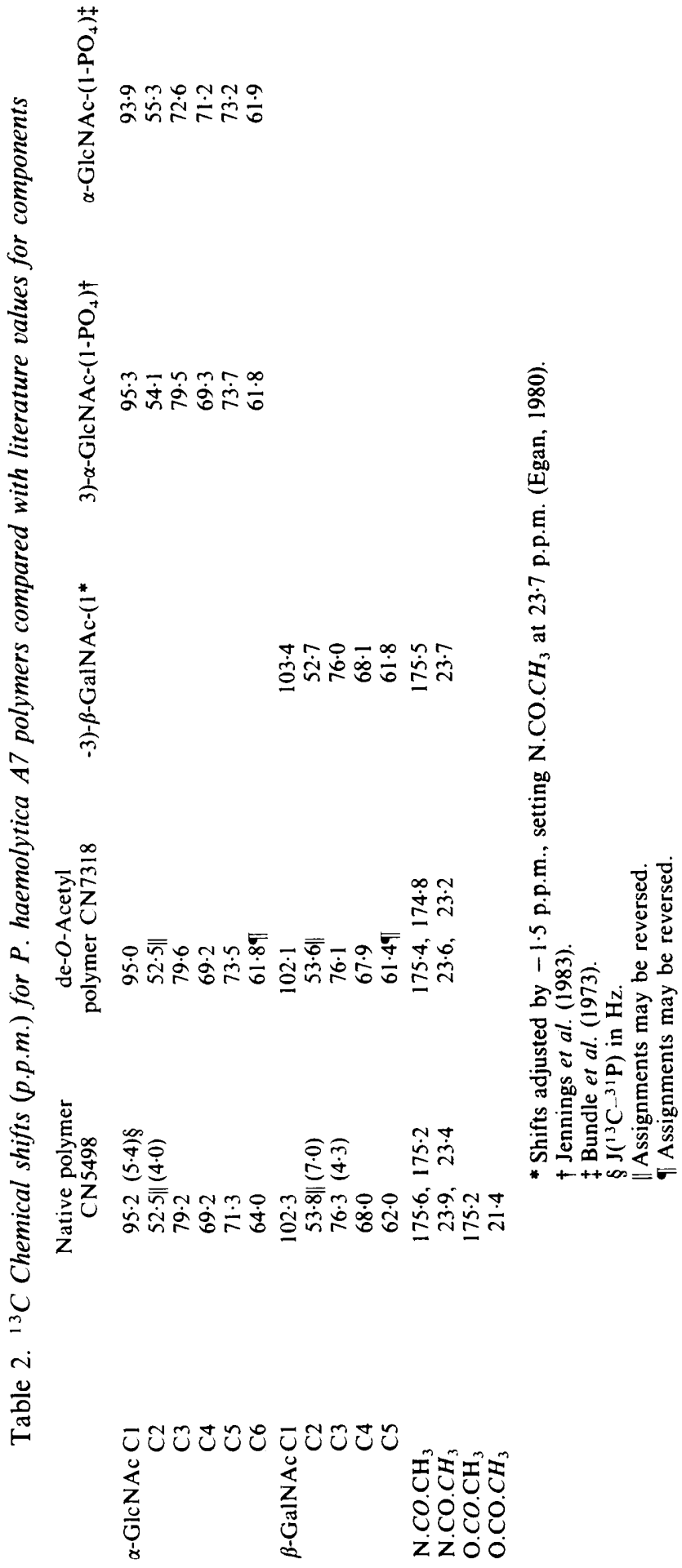


to the de-O-acetylated polymer. Minor lines were clearly seen on the signal of $\mathrm{H} 1 \alpha$-GlcNAc and its connectivity peak in the two-dimensional COSY spectrum (Fig. 3). Also at higher field an extra connectivity peak, its fine structure being $(3 \times 3)$, was compatible with $\mathrm{H} 3$ coupling to $\mathrm{H} 4$, the latter being shifted to higher field in the de- $O$-acetylated $\alpha$-GlcNAc. Minor signals of H5 and H6 of $\alpha$-GlcNAc are expected at higher field than in $O$-acetylated $\alpha$-GlcNAc but they were not located, probably because they occurred in a crowded part of the spectrum, $\sim \delta 3.7$.

\section{Toxicity of purified polymer}

When $5 \mathrm{mg}$ purified polymer (strain $\mathrm{CN} 7318$ ) was injected intravenously into three rabbits, negligible rectal temperature increases were recorded $\left(0 \cdot 3,0.0\right.$ and $\left.0.2^{\circ} \mathrm{C}\right)$. Doses of up to $2.5 \mathrm{mg}$ injected intravenously into chick embryos failed to kill. Limulus titres were also very low, such that in an assay in which an Escherichia coli endotoxin standard sample produced gelation at a level of $8 \mathrm{pg} \mathrm{ml}^{-1}, 3.3 \mathrm{mg} \mathrm{ml}^{-1}$ of the purified polymer was required for gelation to occur. These experiments indicated the absence of endotoxin (lipopolysaccharide) in the purified preparation.

\section{Serological properties}

The purified polysaccharide elicited antibody formation in two sheep when emulsified in oil and administered intraperitoneally.

When the purified polymer was diffused overnight in an immunodiffusion experiment against antiserum raised against $\mathrm{A} 7$ whole cells, a single line of precipitation was observed. This reaction was specific for the $A 7$ serotype and no precipitation was observed against antisera raised against other $\mathrm{A}$ or $\mathrm{T}$ serotype of $P$. haemolytica. In a crossed immunoelectrophoresis experiment (Axelsen et al., 1973) a single defined precipitate arc was produced in the presence of Triton $\mathrm{X}-100$ using this antiserum.

In passive haemagglutination experiments erythrocytes coated with the purified polymer only agglutinated with antisera raised against $P$. haemolytica $\mathrm{A} 7$ whole cells or purified polymer and not against antisera against other $\mathrm{A}$ or $\mathrm{T}$ serotypes. De- $\mathrm{O}$-acetylation of the polymer destroyed both its ability to precipitate with immune serum in immunodiffusion experiments and its ability to absorb on to erythrocytes at neutral $\mathrm{pH}$.

\section{Electron microscopy}

Protein A-gold labelling in conjunction with sheep antiserum raised against the purified polymer was used to demonstrate the peripheral localization of the polysaccharide on the organisms. The appearance of labelled bacteria was identical to those previously published by us for serotype T15 organisms reacted with specific antiserum (Adlam et al., 1985b).

\section{DISCUSSION}

The polymer purified and characterized in the present study was localized on the periphery of the organism. It was shown to be immunogenic and responsible for serotype specificity. Immunological reactivity was apparently dependent on the presence of $O$-acetyl groups without which immune precipitation and erythrocyte absorption properties were lost.

Phosphate-containing polysaccharides in which the phosphate residues are incorporated into the backbone of the polymer have previously been reported as being produced by several other bacteria. Staphylococcus lactis strain 2102 produces, for example, a polymer consisting of 2amino-2-deoxyglucose with phosphate linked into the chain via the $\mathrm{Cl}$ and $\mathrm{C} 6$ residues of the sugars (Archibald \& Stafford, 1972). In this polymer, sugar and phosphate moieties occur in equimolar amounts. A similar polymer but linked $\mathrm{Cl}$ to $\mathrm{C} 4$ is the group specific polysaccharide from Neisseria meningitidis serogroup X (Bundle et al., 1974).

A polymer isolated from Micrococcus sp. Al and related micrococci is similar to the $P$. haemolytica $\mathrm{A} 7$ polymer described in the present study in having two sugar residues for every one phosphate residue (Partridge et al., 1971, 1973). In this case, however, and in the case of a 
polymer from Bacillus subtilis strain 168 (Duckworth et al., 1972) the polymer is a poly(-glucosyl$\mathrm{N}$-acetylgalactosamine 1-phosphate) and the sugar linkage points are different.

Other similar capsular polysaccharides which have been described include those from $N$. meningitidis serogroup L (Jennings et al., 1983) and Haemophilus influenzae type f (BraneforsHelander et al., 1980; Egan et al., 1980) which are both $N$-accetylated hexosamine phosphate polymers.

The authors are grateful to Linda Carpenter and Mr P. A. G. Roberts for technical assistance.

\section{REFERENCES}

Adlam, C., Knights, J. M., Mugridge, A., Lindon, J. C., Baker, P. R. W., Beesley, J. E., Spacey, B., Craig, G. R. \& Nagy, L. K. (1984). Purification, characterization and immunological properties of the serotype-specific capsular polysaccharide of Pasteurella haemolytica (serotype Al) organisms. Journal of General Microbiology 130, 2415-2426.

Adlam, C., Knights, J. M., Mugridge, A., Lindon, J. C. \& Williams, J. M. (1985a). Purification, characterization and immunological properties of the serotype-specific capsular polysaccharide of Pasteurella haemolytica (serotype T4) organisms. Journal of General Microbiology 131, 387-394.

Adlam, C., Knights, J. M., Mugridge, A., Lindon, J. C., Williams, J. M. \& Beesley, J. E. (1985b). Purification, characterization and immunological properties of the capsular polysaccharide of Pasteurella haemolytica (Serotype T15): its identity with the K62 (K2ab) capsular polysaccharide of Escherichia coli and the capsular polysaccharide of Neisseria meningitidis serogroup H. Journal of General Microbiology 131, 1963-1972.

Archibald, A. R. \& Stafford, G. H. (1972). A polymer of $\mathrm{N}$-acetylglucosamine 1-phosphate in the wall of Staphylococcus lactis 2102. Biochemical Journal 130, 681-690.

Axelsen, N. H., Krøll, J. \& Week, B. (1973). A manual of quantitative immunoelectrophoresis. Scandinatian Journal of Immunology 2, Supplement 1, Oslo: Universities Forlaget.

BAX, A. \& FrEEMAN, R. (1981). Investigations of complex networks of spin-spin coupling by two dimensional n.m.r. Journal of Magnetic Resonance 44, 542-561.

Bhattacharjee, A. K., Jennings, H.J., Kenny, C. P., Martin, A. \& SMith, I. C. P. (1975). Structural determination of the sialic acid polysaccharide antigens of Neisseria meningitidis serogroups B and C with carbon 13 nuclear magnetic resonance. Journal of Biological Chemistry 250, 1926-1932.

Bodenhausen, G. \& Freeman, R. (1977). Correlation of proton and carbon-13 spectra by heteronuclear two-dimensional spectroscopy. Journal of Magnetic Resonance 28, 471-476.
Branefors-Helander, P., Kenne, L. \& Lindquist, B. (1980). Structural studies of the capsular antigen of Haemophilus influenzae type f. Carbohydrate Research 79, 308-312.

Bundle, D. R., Jennings, H. J. \& SMith, I. C. P. (1973). The carbon-13 nuclear magnetic resonance spectra of 2-acetamido-2-deoxy-D-hexoses and some specifically deuterated, $O$-acetylated and phosphorylated derivatives. Canadian Journal of Chemistry $\mathbf{5 1}$, 3812-3819.

Bundle, D. R., Jennings, H. J. \& Kenny, C. P. (1974). Studies on the group-specific polysaccharide of Neisseria meningitidis serogroup $\mathrm{X}$ and an improved procedure for its isolation. Journal of Biological Chemistry 15, 4797-4801.

Duckworth, M., Archibald, A. R. \& Baddiley, J. (1972). The location of $N$-acetylgalactosamine in the walls of Bacillus subtilis 168. Biochemical Journal 130 , 691-696.

EGAN, W. (1980). Structure of the capsular polysaccharide antigens from Haemophilus influenzae and Neisseria meningitidis by $\mathrm{C}^{13} \mathrm{nmr}$ spectroscopy. Magnetic Resonance in Biology 1, 197-258.

Egan, W., Tsui, F. P. \& Schneerson, R. (1980). Structural studies of the Haemophilus influenzae type f capsular polysaccharide. Carbohydrate Research 79, 271-277.

Jennings, H. J., Lugoski, C. W., Ashton, F. E. \& RYAN, J. A. (1983). The structure of the capsular polysaccharide obtained from a new serogroup (L) of Neisseria meningitidis. Carbohydrate Research 112. 105-111.

Partridge, M. D., Davison, A. L. \& Baddiley, J. (1971). A polymer of glucose and $N$-acetylgalactosamine 1-phosphate in the wall of Micrococcus sp. Al. Biochemical Journal 121, 695-700.

Partridge, M. D., Davison, A. L. \& Baddiley, J. (1973). The distribution of teichoic acids and sugar 1-phosphate polymers in walls of micrococci. Journal of General Microbiology 74, 169-173.

Stoffyn, P. J. \& JEANLOZ, R. W. (1954). Identification of amino sugars by paper chromatography. Archices of Biochemistry and Biophysics 52, 373-379. 